Onkologe 2013 $\cdot$ 19:98-100

DOI 10.1007/s00761-012-2398-4

Online publiziert: 10. Januar 2013

c) Springer-Verlag Berlin Heidelberg 2013

J. Hübner ${ }^{1}$ I. Adamietz ${ }^{2}$ R. Grützmann ${ }^{3} \cdot$ K. Höffken ${ }^{4}$

${ }^{1}$ Dr. Senckenbergisches Chronomedizinisches Institut, J.W. Goethe-Universität Frankfurt

2 Klinik für Strahlentherapie und Radioonkologie, Marienhospital Herne, Klinikum der Ruhr-Universität Bochum, Herne

${ }^{3}$ Klinik für Viszeral-, Thorax- und Gefäßchirurgie, Universitätsklinikum Carl Gustav Carus, Dresden

${ }^{4}$ Universitätsklinikum Jena

\title{
Von der Kombination aus komplementärer und konventioneller Onkologie zur integrativen Onkologie
}

kräfte eingesetzt werden. Ein gefährlicher Gegensatz zur Kombination komplementärer und schulmedizinischer Maßnahmen (integrative Onkologie) sind alternative Verfahren. Diese sollen als Ersatz für schulmedizinische Maßnahmen gelten und sind aus wissenschaftlicher Sicht abzulehnen. Tumorpatienten informieren sich gegenwärtig zunehmend umfangreicher und besser über ihre Tumorerkrankung und die therapeutischen Möglichkeiten.

\section{》) Die Erwartungen der Patienten werden immer größer} Fundament stehen die Schulmedizin und die komplementäre Medizin. Beide unterscheiden sich nach dem Grad der aktuell vorliegenden Evidenz. Eine Integration, von der wir wesentliche neue Impulse für die Patientenversorgung erwarten können, entsteht nicht durch ihre Kombination. Ein modernes Verständnis des Begriffs „Integrative Onkologie“ stellt die Patientenperspektive (als Zielrichtung der Integration) in den Mittelpunkt. Hierbei sind Kenntnisse der Schulmedizin wie der komplementären Medizin notwendig; im Vordergrund steht aber die Haltung dem Patienten gegenüber.

Im vorliegenden Schwerpunktheft benutzen wir die Bezeichnung Komplementärmedizin als einen Sammelbegriff für ergänzende Heilverfahren, die zur Linderung von verschiedenen Symptomen und zur Förderung der Selbstheilungs-
Der wissenschaftlich begründete und professionelle Optimismus, mit dem die modernen Tumortherapien in den Medien verkündet werden, lässt die Erwartungen der Patienten steigen. Die von den Betroffenen erhofften und auch eingeforderten therapeutischen Ziele werden deshalb ehrgeiziger.

Dennoch wird nach der Lektüre, nach Gesprächen mit betroffenen Bekannten, Angehörigen und nach den ersten Arztgesprächen für die meisten Patienten klar, dass die Tumortherapien unter Umständen eine begrenzte Wirksamkeit haben und dass die Behandlung beschwerlich und mit einigen Folgen belastet sein kann. Deshalb keimt in jedem Patienten der Gedanke auf, die Tumortherapie sinnvoll zu ergänzen. Der Stoff für diese Überlegun- gen wird gerne in Selbsthilfegruppen, den Warteräumen der Arztpraxen und im Familienkreis geliefert. Ihre Quellen sind verschiedene Illustrierte, Internet oder nicht selten Mundpropaganda.

$\mathrm{Zu}$ den komplementären Verfahren gehören zahlreiche Therapien, deren Wirksamkeit nicht durch wissenschaftlich fundierte Methoden überprüft ist. Wichtig für ihren Einsatz unter rationalen Gesichtspunkten ist es, die Sicherheit der Patienten in den Vordergrund zu stellen und z. B. negative Interaktion mit der Schulmedizin auszuschließen. Im Hinblick auf die medizinischen Weiterentwicklungen der Medizin sind Onkologen dafür verantwortlich, dem Patienten das Feld der komplementären Methoden und die damit assoziierten wissenschaftlichen $\mathrm{Ge}$ sichtspunkte darzustellen. Hierbei erreichen Stellungnahmen wie der Hinweis auf die schwache Studienlage, die übrigens manchmal nicht ganz berechtigt ist, nicht aus. Aufgabe der wissenschaftlichen Fachgesellschaften ist eine kritische und konstruktive Auseinandersetzung mit dem Thema.

》) Tumorpatienten befinden sich in einer besonderen Lebenssituation

Der Tumorpatient befindet sich meistens in einer Ausnahmesituation in Anbetracht der Erst- oder Rezidivdiagno- 
Hier steht eine Anzeige.

算 Springer 
se einer Krebserkrankung. Er empfindet eine existenzielle Bedrohung nicht nur wegen der Lebensbedrohlichkeit der Erkrankung, sondern auch dadurch, dass das Vertrauen in die "Zuverlässigkeit“ des eigenen Körpers erschüttert ist. Viele Patienten entwickeln aber in dieser Situation ungeahnte Kräfte und entdecken Ressourcen. Vor dieser Kulisse tauchen häufig am Ende der vertrauensvollen Therapiegespräche mit den Krebspatienten Sätze auf wie: ,... und was könnte ich sonst noch tun?" oder „... können sie mir noch etwas Zusätzliches zu meiner Krebstherapie empfehlen?". Sie sind Ausdruck der Suche nach optimaler, individueller und wirksamer Behandlung, stellen ein Angebot dar, Eigenverantwortung zu übernehmen, zielen aber als Fragen unmittelbar auch auf die Verantwortung des Arztes. Beteiligt sich der Arzt an dieser Diskussion nicht und bleibt den Patienten die Antwort schuldig, erschüttert er das geschaffene Vertrauen, zerstört die für den Erfolg der Therapie so entscheidende positive Arzt-Patienten-Beziehung und treibt den Patienten mit hoher Wahrscheinlichkeit in die Parallelwelt der alternativen Medizin. Deshalb sind ein Achselzucken oder abweisende, inkompetente Antworten zu vermeiden. Da die Nutzung von komplementären Methoden sich seit Jahren relativ stabil auf einige Themen konzentriert, ist es auch für den Onkologen nicht schwierig, sich hier Kenntnisse anzueignen und diese auch zu aktualisieren.

Damit der onkologisch aufklärende Arzt die Probleme der komplementären Medizin besser versteht, werden in diesem Heft verschiedene Themen besprochen und erläutert.

Für die Beratung von Patienten zu KAM in der Onkologie wurden 2012 von Experten der Arbeitsgemeinschaft Prävention und Integrative Onkologie der Deutschen Krebsgesellschaft Empfehlungen entwickelt. Beratungen erfordern nicht nur spezifisches Fachwissen über KAM-Verfahren, sondern auch onkologische Expertise und Kompetenzen in Gesprächsführung und Beratung, die durch entsprechende Weiterbildungen zu erwerben und zu erhalten sind. Dieser Expertenkonsens wird in dem einleitenden Beitrag beschrieben.
Der Artikel „Krebsdiäten“ stellt die Fakten zu seit Jahrzehnten bedeutsamen und aktuell propagierten Ernährungsformen zusammen.

Im Beitrag über alternative Medizin wird auf die unmittelbaren wie indirekten Gefahren dieser Maßnahmen hinweisen. Es werden praktische Tipps und Hinweise formuliert, wie ein falscher Weg dem Patienten erspart werden kann.

Im Artikel „Traditionelle europäische Heilverfahren" werden uralte Heilungsmethoden beschrieben, ihre Einschätzung aus der heutigen Sicht gegeben und die Erklärung ihre Wirksamkeit beleuchtet.

Im Beitrag zur Phytotherapie als eine der Supportivmaßnahmen werden die belegten Erfolge dieser Verfahren beschrieben. Es gibt inzwischen Substanzen, die den Weg in die Schulmedizin gefunden haben, nachdem mit modernen Methoden der Nachweis ihrer Wirksamkeit gelungen ist. Auch sekundäre Pflanzenstoffe sowie weitere Natursubstanzen in der Onkologie werden aufgeführt und ihre nachgewiesene Wirksamkeit eingeschätzt.

Im Kapitel „Nahrungsergänzungsmittel" werden vorhandene wissenschaftliche Daten sowie der theoretische Hintergrund zur Anwendung dieser alimentären Substanzen beschrieben.

Die Herausgeber hoffen, dass sie mit diesem Heft das Wissen der onkologisch tätigen Ärzte sinnvoll erweitern und die schwierigen Aspekte der Therapiegespräche mit den Tumorpatienten auf diese Weise erleichtern und ein gelegentliches Achselzucken der Therapeuten auf die Frage ,... kann ich noch zusätzlich etwas machen?" zu vermeiden helfen.

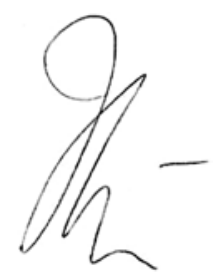

J. Hübner

Für die Herausgeber des Schwerpunktthemas

\section{Hötren}

\section{K. Höffken}

Für die Herausgeber

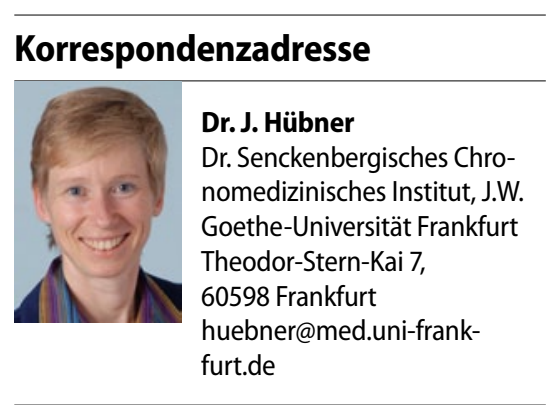

